\title{
Characterisation of Martian Soil Simulants for the ExoMars rover testbed
}

\author{
Christopher Brunskilla,*, Nildeep Patel ${ }^{\mathrm{b}}$, Thibault P. Gouache ${ }^{\mathrm{a}, \mathrm{c}}$, Gregory P. Scott ${ }^{\mathrm{d}}$, \\ Chakravarthini M. Saaj ${ }^{\mathrm{a}}$, Marcus Matthews ${ }^{\mathrm{a}}$, Liang Cui ${ }^{\mathrm{a}}$ \\ ${ }^{a}$ University of Surrey, Surrey Space Centre, Guildford GU2 7XH, United-Kingdom \\ ${ }^{b}$ EADS Astrium Ltd., Gunnels Wood Road, Stevenage, Herts, SG1 2AS, UK \\ ${ }^{c}$ Universit de Toulouse, IGM, ISAE, DMSM, 10 Avenue Edouard Belin, Toulouse 31000, France \\ ${ }^{d}$ AstroTechnic Solutions, Springfield VA, 22152, USA
}

\begin{abstract}
The ESA ExoMars mission involves landing a rover on the surface of Mars on an exobiology mission to extend the search for life. The locomotion capabilities of the ExoMars rover will enable it to use its scientific instruments in a wide variety of locations. Before it is sent to Mars, this locomotion system must be tested and its performance limitations understood. To test the locomotion performance of the ExoMars rover, three Martian regolith simulants were selected: a fine dust analogue, a fine Aeolian sand analogue, and a coarse sand analogue. To predict the performance of the ExoMars rover locomotion system in these three regolith simulants, it is necessary to measure some fundamental macroscopic properties of the materials: cohesion, friction angle, and various bearing capacity constants. This paper presents the tests conducted to determine these properties. During these tests, emphasis was placed on preparing the regolith simulants at different levels of density in order to also evaluate the impact of density on the value of the parameters in particular. It was shown that compaction can influence the Bekker coefficients of pressure-sinkage. The shear properties are consistent with the critical state model at normal stresses similar
\end{abstract}

\footnotetext{
${ }^{*}$ Corresponding author

Email address: c.brunskill@surrey .ac.uk (Christopher Brunskill)
} 
to those of the ExoMars rover in all but one of the simulants, which showed behaviour more consistent with transitional soil behaviour. It is necessary to give due consideration to these variations to ensure a robust test regime is developed when testing the tractive ability of the ExoMars mobility system.

Keywords: Mars, ExoMars, rover, mobility, simulant, soil mechanics

\section{Introduction}

Before a mission is launched to explore the surface of Mars or any other extra-terrestrial body, it is essential to test the performance of all of its subsystems. For a mission like ExoMars, the performance of the rover locomotion system is key to the quantity and diversity of scientific data collected [1]. The locomotion system of ExoMars must thus be tested.

The surface of Mars is covered by regolith: dust, sand and rocks. The terms "soil" and "regolith" are often used interchangeably in this field, and also in this article. Clearly, the most accurate results for performance assessment of a rover would be to trial it on a system testbed filled with substantial bulk of Martian regolith. However, no Martian regolith has been returned to Earth to date, making it necessary to find an Earth-based material analogue which mimics the mechanical properties of these soils that were determined during previous Mars missions. The broad natural variation in the physical composition of Martian regoliths lead to the classification of three common terrain types that a rover, such as ExoMars, would be expected to traverse. These are defined as follows:

- Fine dust

- Fine aeolian sand

- Coarse sand 


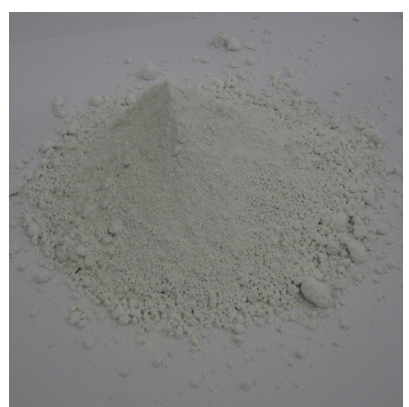

(a) ES-1

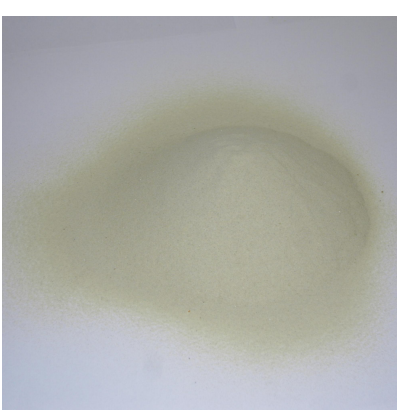

(b) ES-2

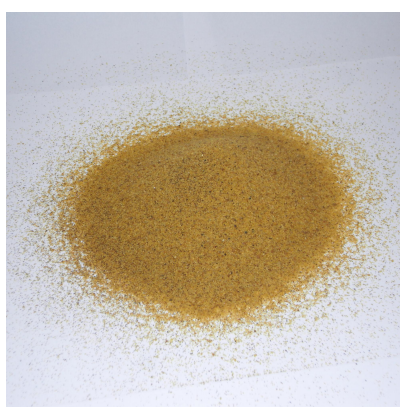

(c) ES-3

Figure 1: Samples of Martian soil simulants

Three materials have been selected to fulfil these requirements. These are classified as sandy or silty soils due to the fine particle sizes involved [2]. The simulants based on these materials are named Engineering Soil Simulant-1 (ES-1), Engineering Soil Simulant-2 (ES-2) and Engineering Soil Simulant-3 (ES-3), comprised from Nepheline Stjernoy 7, Red Hill 110, and Leighton Buzzard DA 30 respectively. All three materials are easily available as off-the-shelf materials at local suppliers and have been procured in quantities of approximately 1 tonne. A full description of these materials and their microscopic properties is provided in a separate publication . Samples of each material are shown in Figure 1 and their characteristic particle size distributions in Figure 2.

Existing performance prediction models for traditional robotic systems (and other models such as those used for cone penetration tests [3]) are based on the macroscopic properties of these soils, such as shear strength. The characteristics of the particles such as shape, density and size distribution that influence the behaviour of the soil mass are rarely considered [4]. Though there is a strong link between the mechanical properties of soil and the microscopic properties of the individual soil particles, there is still much work to be done to develop a widely accepted analytical model to relate one to the other [5], 


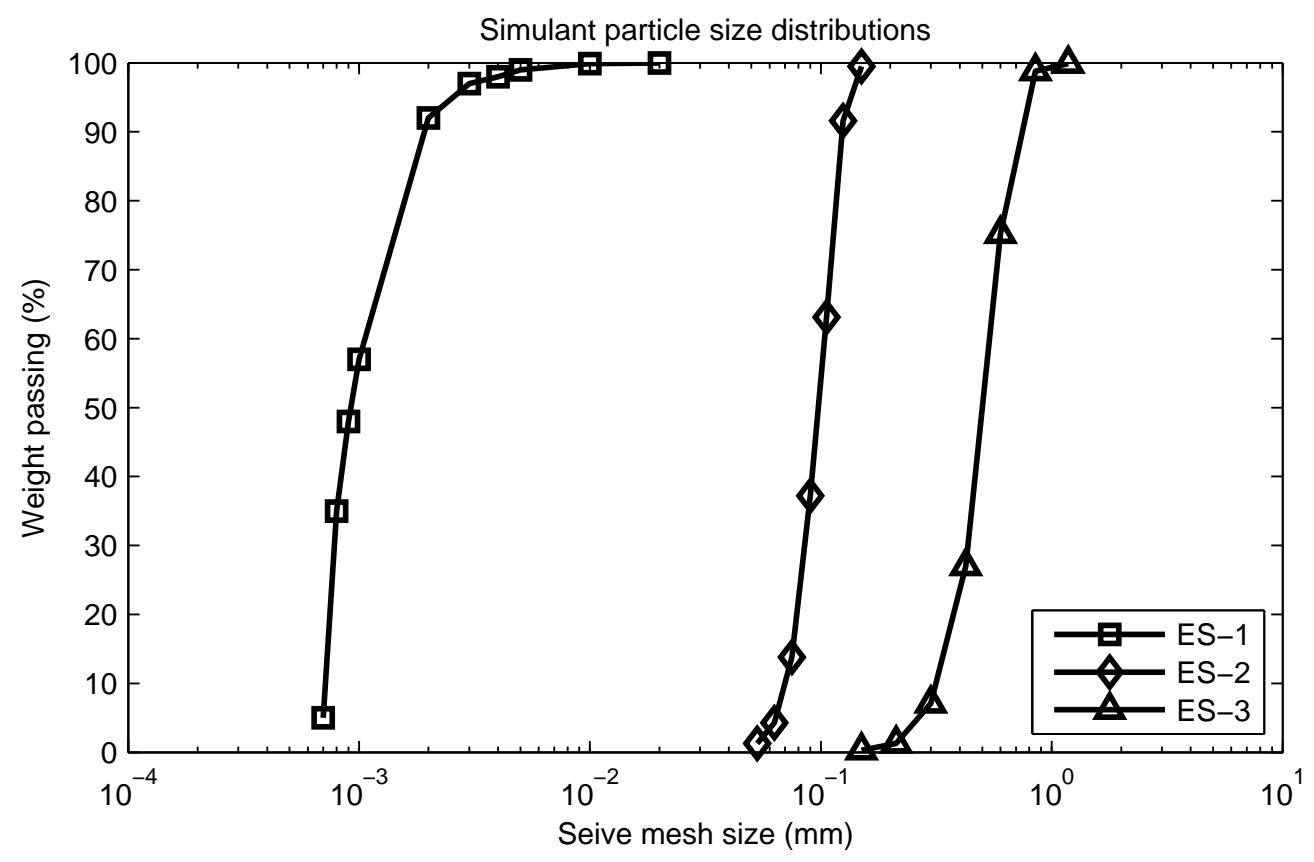

Figure 2: Characteristic particle size distributions for Martian soil simulants ES-1, ES-2 and ES-3

[6]. Therefore, while measurements of the physical parameters allow us to characterise the regolith simulants, they are not sufficient to provide information on the mechanical behaviour.

The macroscopic properties in classical soil mechanics are cohesion, $C$ and angle of internal friction, $\phi$, defining the shear strength of a material. Properties including the sinkage coefficients of cohesion and friction, $k_{C}$ and $k_{\phi}$ respectively, and the sinkage exponent, $n$, are used in the field of terramechanics, a widely used method for modelling vehicle traction performance. Together, these parameters can be used to assess the ability of a terrain to support the traversal of a given vehicle.

Numerous test methods are available to determine these parameters. In this research the direct shear testing (DST) and pressure sinkage testing (PST) methods were selected. 
The DST provides a simple method of testing soil strength along a known shear plane [7] and provides values for $C$ and $\phi$, related by the Mohr-Coulomb expression for soil shear strength

$$
\tau=C+\sigma \tan \phi
$$

Where $\sigma$ is the normal stress applied to the soil. The measured resistance to shearing, $\tau$, during the DST test takes one of two general forms, illustrated in Figure 3 . In the case of vehicle wheels or tracks the residual (or critical state) shear stress is an appropriate measure of the "worst case" strength of a terrain. Based on the available information on state of Martian soils, the tests described in this paper were required to be carried out on dry samples of varying densities. The DST method allowed these parameters to be more easily controlled over than in alternative methods.

The PST is a method proposed by Bekker [8] and further refined by Wong [9]. It is used to obtain the remaining soil parameters by empirical measurement of the ground pressure during sinkage. These parameters are related by the following equation:

$$
p=\left(\frac{k_{C}}{b}+k_{\phi}\right) \cdot z^{n}
$$

Where $b$ is the width or diameter of the pressure plate used. The tests described here were performed on the three simulant materials, selected due to both their particle size ranges and their mineralogy to replicate the regolith types identified above. Other Martian regolith simulants [10] [11] [12], Lunar regolith simulants [13] and Lunar regolith from Apollo missions [14] [15] have also been subject to DST. PST on regolith simulants are reported in [16].

For a given soil the density at which it is tested will have an impact on its mechanical properties. This has been observed through tests such as the DST [17] [18], in-situ on the lunar surface [19] [20], in wheel-soil interaction [21] [4], in leg-soil interaction [12], in 

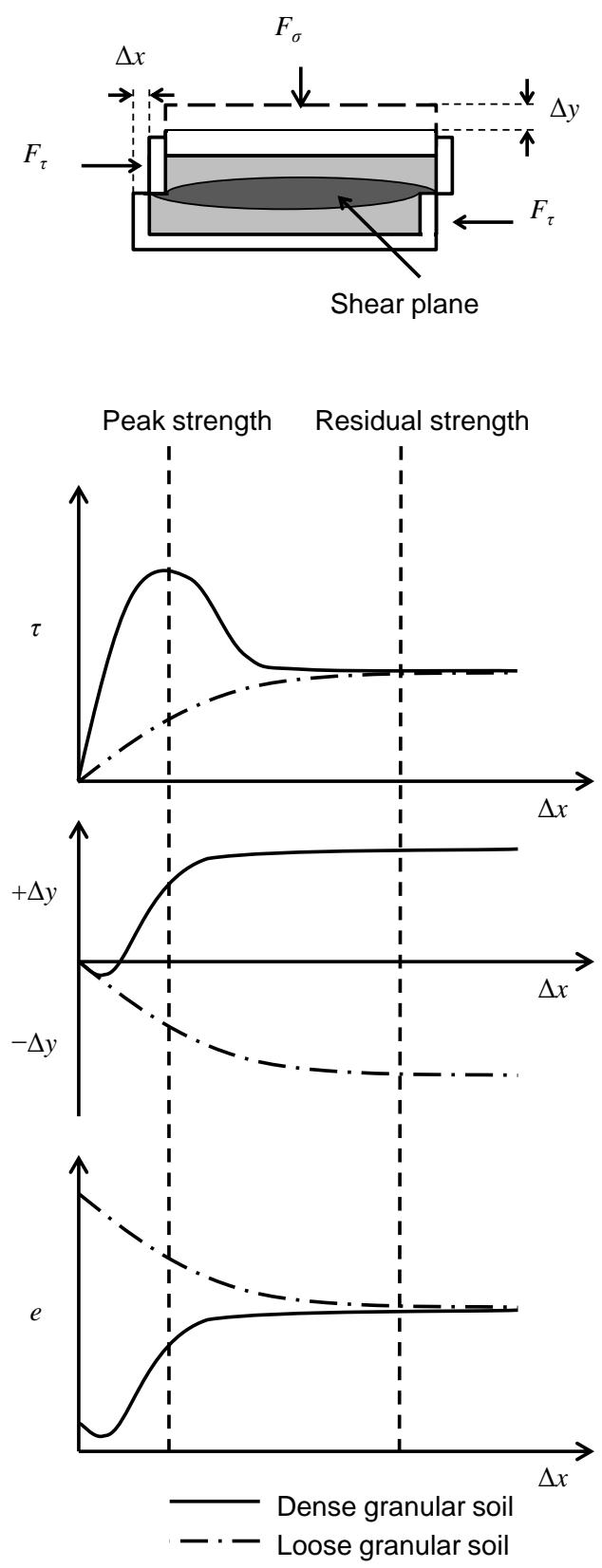

Figure 3: Schematic of a DST shear box and the resulting stress $\tau$, vertical displacement $y$ and void ratio $e$ 
static penetration of soil by a regolith probe [22] [23] and in bio-inspired dual reciprocating drilling into regolith [24]. The recent difficulties of the NASA rover Spirit on Mars are most probably due to the loose compaction of the regolith where it is now trapped [25]. Such a low density regolith will be very challenging to traverse for any small vehicle. As such, the DST and PST simulant test samples were prepared at three different densities. This allows the variation in the mechanical properties of the three regolith simulants to be studied. Moreover, the impact of density on the performance of a rover locomotion system, such as that used on ExoMars, can be assessed.

\section{Mechanical testing}

\subsection{Humidity content}

Like density, the moisture content of loose sand can also significantly affect the mechanical parameters [26]. The simulants were stored in air-tight transportation drums and tested in climate-controlled laboratories. The moisture content of each simulant was determined using the ASTM D2216-05 standard [27]. Each of the three materials were tested shortly after delivery, after allowing time to acclimate to the laboratory conditions. Further moisture measurements were completed during the DST and PST experiments with samples taken at the same time as the test material. Multiple samples were collected, weighed, and dried in an oven for 48 hours at $120^{\circ} \mathrm{C}$. The difference in mass was recorded to determine the moisture content as a percentage of dry mass.

The initial tests were carried out in a laboratory with a temperature of $21.5^{\circ} \mathrm{C}$ and humidity of $31 \%$. The DST was carried out on each material in the same laboratory, at this time the average temperature was $19^{\circ} \mathrm{C}$ and average humidity $39 \%$. The PST was carried

out in an alternative laboratory, where the average temperature was $17.5^{\circ} \mathrm{C}$ and average humidity $38 \%$. Table 1 summarises the moisture content measurements for each simulant 


\begin{tabular}{l|ccc} 
& On arrival (\%) & DST (\%) & PST (\%) \\
\hline \hline ES-1 & & & \\
Moisture & 0.35 & 0.30 & 0.29 \\
Std. dev. & 0.04 & 0.07 & 0.02 \\
ES-2 & & & \\
Moisture & 0.20 & 0.17 & - \\
Std. dev. & 0.03 & 0.05 & - \\
ES-3 & & & \\
Moisture & 0.34 & 0.22 & 0.19 \\
Std. dev. & 0.05 & 0.01 & 0.04
\end{tabular}

Table 1: Average moisture content of each simulant. (NB. No PST was carried out on ES-2 due to insufficient quantity)

in each case. The materials were procured as "dry", as necessitated by the requirement to replicate the Martian conditions. The moisture content readings confirmed this assumption, allowing each of the materials to also be considered dry.

\subsection{Direct Shear Testing (DST)}

\subsubsection{Method}

Three methods were used to prepare the DST samples to achieve the desired variation in density: pouring from a small height, pouring from higher distance and pressurecompaction. The pouring techniques have been used previously to validate the preparation of soil samples in $30 \mathrm{~L}$ soil bins and $250 \mathrm{~mL}$ cups, discussed in [28]. These methods were tested in the direct shear apparatus and have been shown to give repeatable results in each case. The pressure-compaction technique has also been shown in [29] to provide repeatable results in DST preparation. 
Pouring simulant from a low height is used to prepare samples with high void ratios, providing a low density. The method requires pouring of the material at a constant rate into the shear box from the lowest achievable height. For the equipment used here this was $10 \mathrm{~cm}$ above the top of the shear box. The medium density samples were prepared by pouring from a height of sufficient magnitude to allow the soil grains to achieve their terminal velocity, at which point the flow is fully turbulent. Previous experimental studies on this technique have shown that a height of $40 \mathrm{~cm}$ or above is sufficient for granular materials [30]. As such, a height of $50 \mathrm{~cm}$ above the top of the shear box was used when preparing these samples.

High density samples were prepared with a method referred to as pressure-compaction. This technique provides much higher densities, typically over 80\% [29]. The method requires the DST shear box to be filled in three stages, each layer being compacted by imposing a static pressure and manual vibration before filling with the next.

Each low and medium density sample was prepared in place in the DST apparatus to avoid unwanted disturbances to the delicate particle structure, the result of which would be a change in the sample density. To determine the density of the low and medium density samples, calibration runs using the respective pouring methods were performed on the bench prior to the DST experiments. Once filled, the sample height in the shear box was measured to obtain the volume. The complete shear box was then weighed and the sample density obtained by subtracting the empty shear box weight. These values were then assumed for each test using the respective preparation methods. As the high density samples were already compressed outside of the main apparatus they were not sensitive to such disturbances and could be measured and weighed before each test.

The resulting densities and the respective void ratios are presented in Table 2. In each case, variation in the preparation method creates sufficient variation in samples density, with a difference of nearly $0.2 \mathrm{~g} / \mathrm{cm}^{3}$ in the case of ES-2 and ES-3 and nearly $0.7 \mathrm{~g} / \mathrm{cm}^{3}$ 
for ES-1. More importantly, the data have a standard deviation low enough to assume a reasonably compact spread in density.

The tests were completed following the ASTM D3080-04 standard with a minor modification; porous inserts were not used as all simulants are dry. Figure 4 shows the ELE apparatus used for this set of tests. Considering the estimated mass for ExoMars rover breadboard, it was assumed that the target rover mass is approximately $200 \mathrm{~kg}$. This would produce an average mass of $33.3 \mathrm{~kg}$ distributed across 6 wheels or $66.6 \mathrm{~kg}$ across 3 wheels, if reducing the distribution due to wheel walking. Considering the average wheel footprint of $11 \mathrm{~cm}$ x $11 \mathrm{~cm}$, the pressure distribution in Martian gravity $\left(3.69 \mathrm{~m} / \mathrm{s}^{2}\right)$ would be $10.2 \mathrm{kPa}$ or $20.3 \mathrm{kPa}$, respectively. Using a standard $6 \mathrm{~cm} \times 6 \mathrm{~cm}$ direct shear box, the required masses will range from $3.7 \mathrm{~kg}$ to $7.4 \mathrm{~kg}$. Therefore, a custom low mass hanger with a mass of $0.58 \mathrm{~kg}$ and top cap with a mass of $0.54 \mathrm{~kg}$ were used. Three additional hanging masses, $2 \mathrm{~kg}, 5 \mathrm{~kg}$ and $8 \mathrm{~kg}$, were selected to provide a regular distribution of normal stress across the required range. The resulting normal stresses are $8.7 \mathrm{kPa}, 17.1$ $\mathrm{kPa}$ and $25.5 \mathrm{kPa}$, referred to as low, medium and high pressure, respectively.

\subsubsection{Results}

Each test was repeated to provide a minimum of three consistent results. The final shear stresses are measured at the critical state, where the stress is constant, and averaged for each normal pressure. The friction angles and cohesion are shown for ES-1 in Table 3 , ES-2 in Table 4 and ES-3 in Table 5 . Here, analysis of the failure envelopes has been completed using both the tangent method (or instantaneous yield loci) and the secant method (or effective yield loci) [31]. Examples of each are shown in Figure 5. The secant method of analysis for the DST, which assumes zero cohesion for each normal stress, will find a range of internal friction angles for each relationship [32]. When a value for cohesion is desired, the tangential method is used. In this case, a linear fit is found for the critical state 


\begin{tabular}{|c|c|c|c|c|}
\hline & $\begin{array}{l}\text { Particle } \\
\text { density } \\
\left(\mathrm{g} / \mathrm{cm}^{3}\right)\end{array}$ & $\begin{array}{l}\text { Low } \\
\text { density } \\
\left(\mathrm{g} / \mathrm{cm}^{3}\right)\end{array}$ & $\begin{array}{l}\text { Medium } \\
\text { density } \\
\left(\mathrm{g} / \mathrm{cm}^{3}\right)\end{array}$ & $\begin{array}{l}\text { High } \\
\text { density } \\
\left(\mathrm{g} / \mathrm{cm}^{3}\right)\end{array}$ \\
\hline \multicolumn{5}{|l|}{ ES-1 } \\
\hline Density & 2.32 & 0.54 & 0.79 & 1.21 \\
\hline Std. dev. & - & 0.017 & 0.018 & 0.015 \\
\hline Void ratio $(e)$ & - & 3.30 & 1.93 & 0.91 \\
\hline \multicolumn{5}{|l|}{ ES-2 } \\
\hline Density & 2.56 & 1.24 & 1.36 & 1.44 \\
\hline Std. dev. & - & 0.004 & 0.014 & 0.018 \\
\hline Void ratio $(e)$ & - & 1.06 & 0.88 & 0.78 \\
\hline \multicolumn{5}{|l|}{ ES-3 } \\
\hline Density & 2.60 & 1.46 & 1.58 & 1.64 \\
\hline Std. dev. & - & 0.004 & 0.006 & 0.029 \\
\hline Void ratio $(e)$ & - & 0.78 & 0.64 & 0.58 \\
\hline
\end{tabular}

Table 2: Average density of each material when prepared using low, medium and high density sample preparation techniques for direct shear testing. 


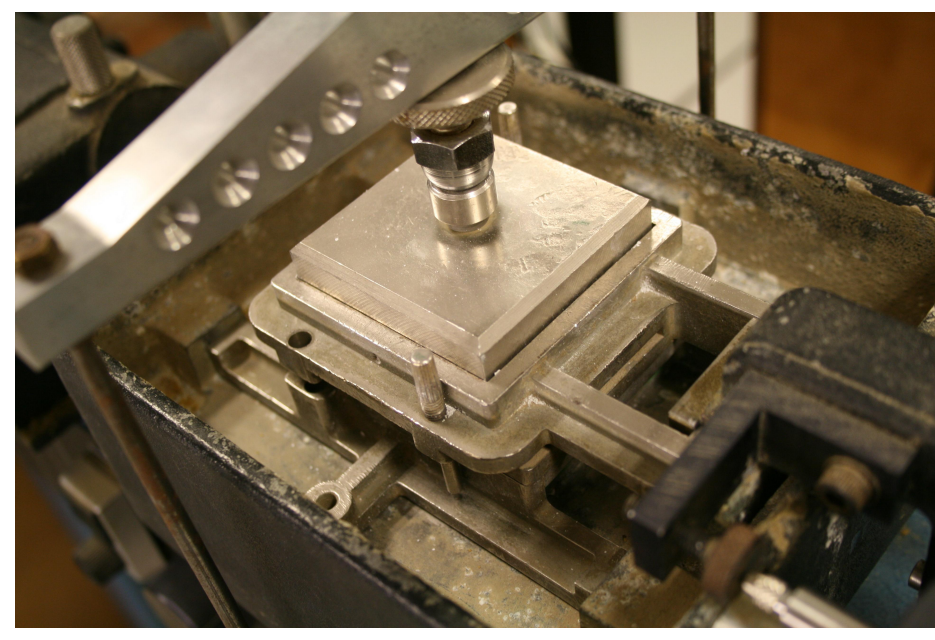

(a) The shear box top cap supports the mass hanger, distributing the pressure over the entire sample surface.

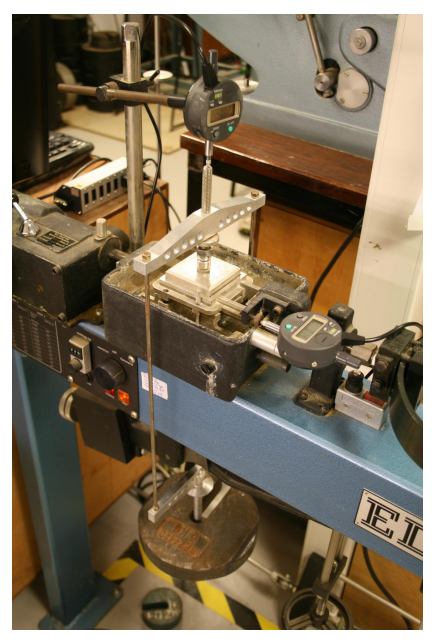

(b) A lightweight hanger and $5 \mathrm{~kg}$ mass can be seen in place over the shear box.

Figure 4: DST Apparatus

shear stress points at all normal stresses. Where this line intersects the y-axis determines the cohesion in Pascals, while the angle of the line determines the angle of internal friction. However, the failure envelope is not strictly linear. As normal stress tends to zero, the cohesion also has to tend to zero to ensure an intercept at the origin with zero normal stress. At very low normal stresses this results in an increasingly higher gradient in the fit line, increasing the friction angle and reducing the cohesion.

Simulant ES-1 is the only of the three soils to consistently show significant cohesion at all compaction levels. While the instantaneous friction angle remains consistent, this does result in variation in the effective friction angles. It is also the only material to show notable difference in the cohesion as density is varied. As such, extremely fine materials of this nature are likely to cause the greatest problems when assessing their trafficability for rovers. 


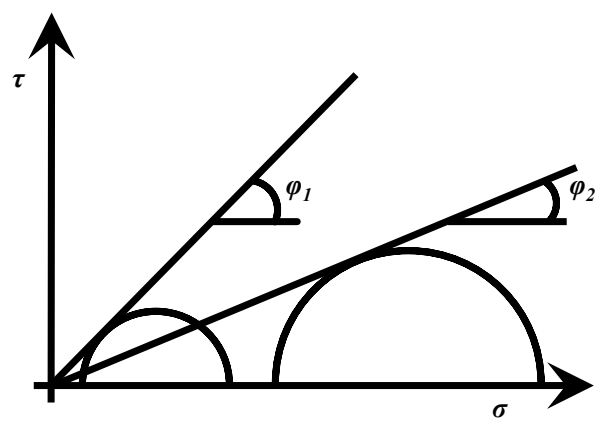

(a) Secant form of failure envelope

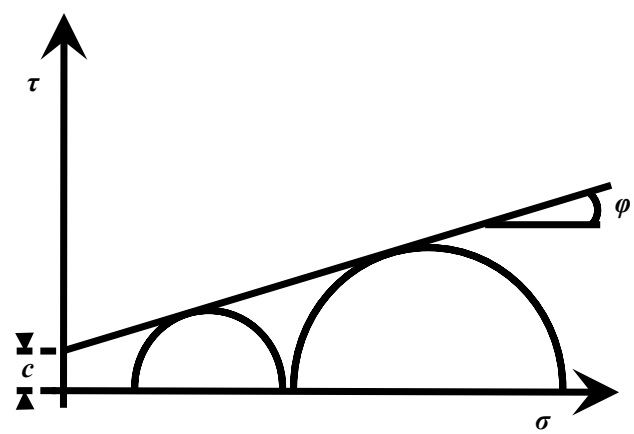

(b) Tangent form of failure envelope

Figure 5: Shear strength failure envelopes

The results for the ES-2 tests show that the instantaneous and effective friction angles are similar in all density cases. This would imply the material would be expected to have an extremely low cohesion. This would explain the negative cohesion observed in the low and high density tests, where even a small error margin would offset the linear fit trend line. The tests on ES-3 also show little variation in the instantaneous and effective values of friction angle, also implying a very low cohesion. An exception to this is observed in the high density tests, where a slightly higher cohesion has produced a lower instantaneous friction angle. It is likely that the more robust preparation method causes the breakdown of any metastable particle structure which may result from the less disruptive methods used to prepare the low and medium density samples.

Typical plots for each simulant and at high normal pressure are shown in Figure 6. Figure 7 shows the failure envelopes for each soil at each density. 


\begin{tabular}{l|lllll} 
Density $\left(\mathrm{g} / \mathrm{cm}^{3}\right)$ & Normal & $\begin{array}{l}\text { Shear stress } \\
(\text { critical }\end{array}$ & $\begin{array}{l}\text { Effective } \\
\text { friction } \\
\text { pressure }\end{array}$ & $\begin{array}{l}\text { Instant. } \\
\text { friction } \\
\text { angle }\left(^{\circ}\right)\end{array}$ & $\begin{array}{l}\text { Instant. co- } \\
\text { angle }\left(^{\circ}\right)\end{array}$ \\
\hline \hline \multirow{3}{*}{ Low dension $(\mathrm{kPa})$}
\end{tabular}

Table 3: ES-1 soil simulant direct shear results 


\begin{tabular}{|c|c|c|c|c|c|}
\hline Density $\left(\mathrm{g} / \mathrm{cm}^{3}\right)$ & $\begin{array}{l}\text { Normal } \\
\text { pressure } \\
(\mathrm{kPa})\end{array}$ & $\begin{array}{l}\text { Shear stress } \\
\text { (critical } \\
\text { state) }(\mathrm{N})\end{array}$ & $\begin{array}{l}\text { Effective } \\
\text { friction } \\
\text { angle }\left(^{\circ}\right)\end{array}$ & $\begin{array}{l}\text { Instant. } \\
\text { friction } \\
\text { angle }\left(^{\circ}\right)\end{array}$ & $\begin{array}{l}\text { Instant. co- } \\
\text { hesion }(\mathrm{kPa})\end{array}$ \\
\hline \multirow{3}{*}{ Low density } & 8.70 & 6.76 & 37.84 & \multirow{3}{*}{38.16} & \multirow{3}{*}{-0.24} \\
\hline & 17.10 & 12.85 & 36.94 & & \\
\hline & 25.50 & 19.96 & 38.05 & & \\
\hline \multirow{3}{*}{ Medium density } & 8.70 & 7.77 & 41.73 & \multirow{3}{*}{38.33} & \multirow{3}{*}{0.64} \\
\hline & 17.10 & 13.65 & 38.60 & & \\
\hline & 25.50 & 21.04 & 39.52 & & \\
\hline \multirow{3}{*}{ High density } & 8.70 & 7.44 & 40.54 & \multirow{3}{*}{41.43} & \multirow{3}{*}{-0.82} \\
\hline & 17.10 & 13.08 & 37.42 & & \\
\hline & 25.50 & 22.26 & 41.12 & & \\
\hline
\end{tabular}

Table 4: ES-2 soil simulant direct shear results 


\begin{tabular}{|c|c|c|c|c|c|}
\hline Density $\left(\mathrm{g} / \mathrm{cm}^{3}\right)$ & $\begin{array}{l}\text { Normal } \\
\text { pressure } \\
(\mathrm{kPa})\end{array}$ & $\begin{array}{l}\text { Shear stress } \\
\text { (critical } \\
\text { state) }(\mathrm{N})\end{array}$ & $\begin{array}{l}\text { Effective } \\
\text { friction } \\
\text { angle }\left(^{\circ}\right)\end{array}$ & $\begin{array}{l}\text { Instant. } \\
\text { friction } \\
\text { angle }\left(^{\circ}\right)\end{array}$ & $\begin{array}{l}\text { Instant. co- } \\
\text { hesion }(\mathrm{kPa})\end{array}$ \\
\hline \multirow{3}{*}{ Low density } & 8.70 & 6.71 & 37.62 & \multirow{3}{*}{35.76} & \multirow{3}{*}{0.26} \\
\hline & 17.10 & 12.20 & 35.49 & & \\
\hline & 25.50 & 18.80 & 36.41 & & \\
\hline \multirow{3}{*}{ Medium density } & 8.70 & 6.86 & 38.17 & \multirow{3}{*}{37.60} & \multirow{3}{*}{0.25} \\
\hline & 17.10 & 13.60 & 38.50 & & \\
\hline & 25.50 & 19.79 & 37.82 & & \\
\hline \multirow{3}{*}{ High density } & 8.70 & 7.59 & 41.08 & \multirow{3}{*}{34.31} & \multirow{3}{*}{1.37} \\
\hline & 17.10 & 12.48 & 36.13 & & \\
\hline & 25.50 & 19.05 & 36.76 & & \\
\hline
\end{tabular}

Table 5: ES-3 soil simulant direct shear results 


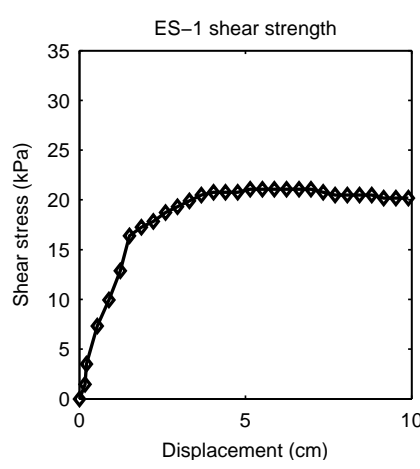

(a) ES-1

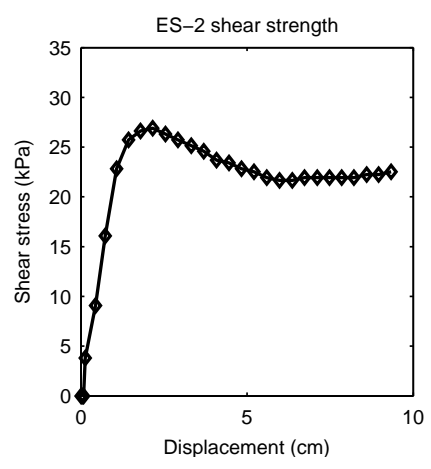

(b) ES-2

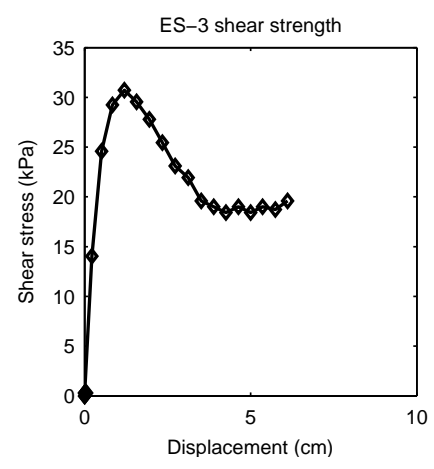

(c) ES-3

Figure 6: Typical shear stress versus displacement curves obtained during DST of ES-1, ES-2 and ES-3

\subsection{Pressure Sinkage Testing (PST)}

\subsubsection{Method}

The aim of a PST is to replicate the contact and surface pressure characteristics of a vehicle's mobility system on the terrain under testing. In terrestrial testing this is performed on the terrain in-situ using an instrument such as a Bevameter [9], either using the vehicle itself or one similar in design and weight. For the purposes of these experiments the terrain has been simulated in the laboratory. Instead of a vehicle mounted Bevametertype instrument, a hydraulic bench-top ram, secured to the floor, is used to apply surface pressure to the soil surface. The stroke length of the ram is $20 \mathrm{~cm}$, enough to penetrate to soil depths equal to and greater than half an ExoMars wheel diameter. Each test requires a sample to be tested with a pair of disc shaped pressure plates. These are $5 \mathrm{~cm}$ and 12.5 $\mathrm{cm}$ in diameter, similar in contact area to that ExoMars is expected to produce. The plates were sunk into each sample by manual pumping of the ram, with each pump providing a few millimetres of sinkage; this was completed at a rate of approximately $2 \mathrm{~cm}$ a minute Pressure readings were taken using a digitally recorded proving ring load cell and sinkage 


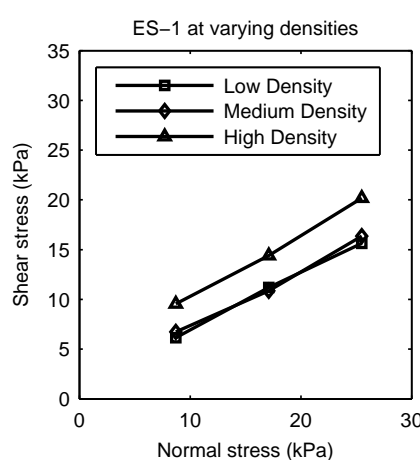

(a) ES-1

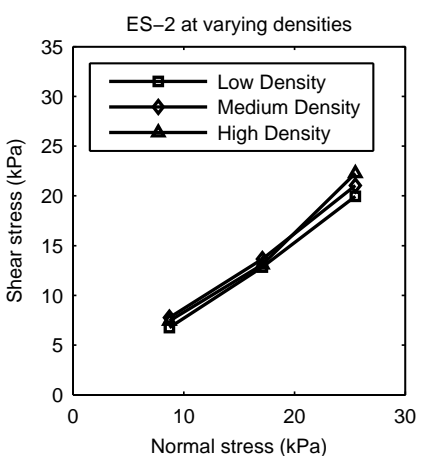

(b) ES-2

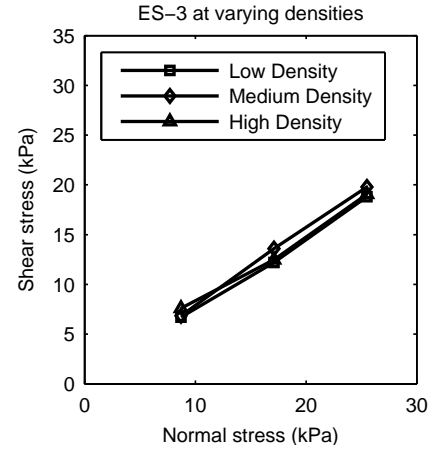

(c) ES-3

Figure 7: The failure envelopes of ES-1, ES-2 and ES-3, following direct shear testing.

measured using a Qualisys motion capture system, tracking a single marker mounted on the ram shaft. Together these data provided the pressure-sinkage results required for analysis of the loading response of each simulant, providing values for coefficient of cohesion $k_{C}$, coefficient of angle of internal friction $k_{\phi}$, and soil exponent $n$.

To further simulate the terrain of the Martian surface, much larger quantities of the simulant materials were used than in the DST experiments. In [29], a method is presented to determine the maximum depth reached by the shear zone activated in the soil by the pressure plate. Considering the bulb of pressure within the soil, the pressure distributed vertically through soil from a square pressure plate shrinks exponentially as depth increases [33]. It can be estimated that $8 \%$ of the applied pressure will be expected at a soil depth twice that of the width of the plate (and $4 \%$ at a depth of three times the width). Considering the maximum stroke length of the ram $(200 \mathrm{~mm})$ and the largest plate dimension $(125 \mathrm{~mm})$, only $4 \%$ of the applied pressure would be expected at $57 \mathrm{~cm}$ from the surface. Therefore, a sample depth of at least $60 \mathrm{~cm}$ would allow each test to have minimal interference of the forces on the drum walls. This depth required much greater quantities of simulant material for each test sample than that used in the DST. Modified 
storage drums were selected for this purpose to allow samples to be prepared to these depths. Specifically, for ES-3, a $205 \mathrm{~L}$ steel drum $89 \mathrm{~cm}$ high and $59 \mathrm{~cm}$ in diameter was used and for ES-1 a $64 \mathrm{~L}$ hard plastic bin measuring $53 \mathrm{~cm}$ high by $43 \mathrm{~cm}$ in diameter (the $7 \mathrm{~cm}$ shortfall in depth can be adjusted for by using no more than $13 \mathrm{~cm}$ of the total ram stroke length). When full, these containers held approximately $300-500 \mathrm{~kg}$ of simulant. Due to a processing complication, insufficient quantity of ES-2 was manufacturedto perform the PST, thus no tests or density measurements were conducted for ES2.

Despite the much larger scale, the preparation methods used in the PST are similar to those used in the DST. The pouring technique makes use of a crane to lift and pour the sand from one drum into another at a constant rate, achieved by the addition of a resealable valve to the drum base. The height is above the required $50 \mathrm{~cm}$ to achieve terminal velocity in the flow, and in this case provided the lowest density sample. To obtain high density samples a vibration technique is used. An off-set motor attached to a $50 \mathrm{~cm}$ square steel plate base supports the sample drum and vibrates the container as the soil is poured. Once the drum is full, the vibration platform is turned off. An intermediate compaction, the medium density, is achieved by preparation using the pouring method above to obtain a low density sample. This is then vibrated for 10 seconds to settle the material. The apparatus set up is shown in Figure 8a.

The very small particle size of the Nepheline powder (material used for ES-1) presented a health and safety issue due to the large quantities of dust left suspended in the atmosphere. Therefore, to reduce the quantity of dust generated by the preparation of this simulant, it was tested in the $64 \mathrm{~L}$ hard plastic bin instead of the $205 \mathrm{~L}$ steel drums. The same three sample preparation techniques were used, however, the simulant was manually poured into the $64 \mathrm{~L}$ bin using a smaller container. As with the DST samples, these preparation methods follow the same methodology as validated in [28].

The ES-1 and ES-3 sample densities were measured using both a volumetric mea- 


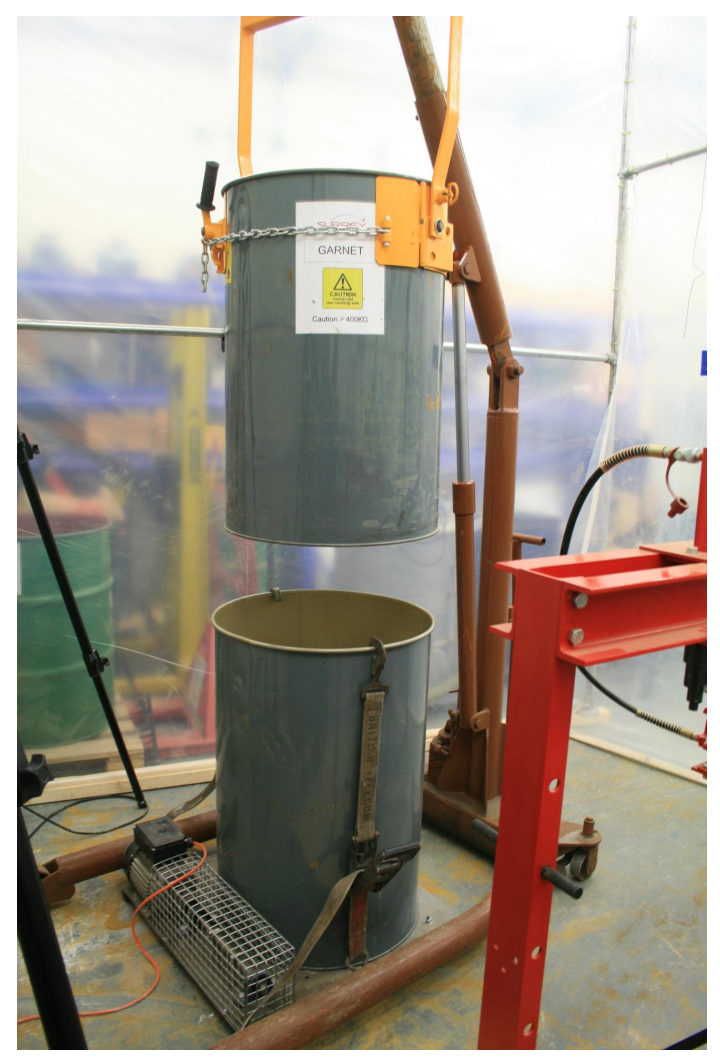

(a) Two $205 \mathrm{~L}$ drums are shown in preparation for pouring a material sample

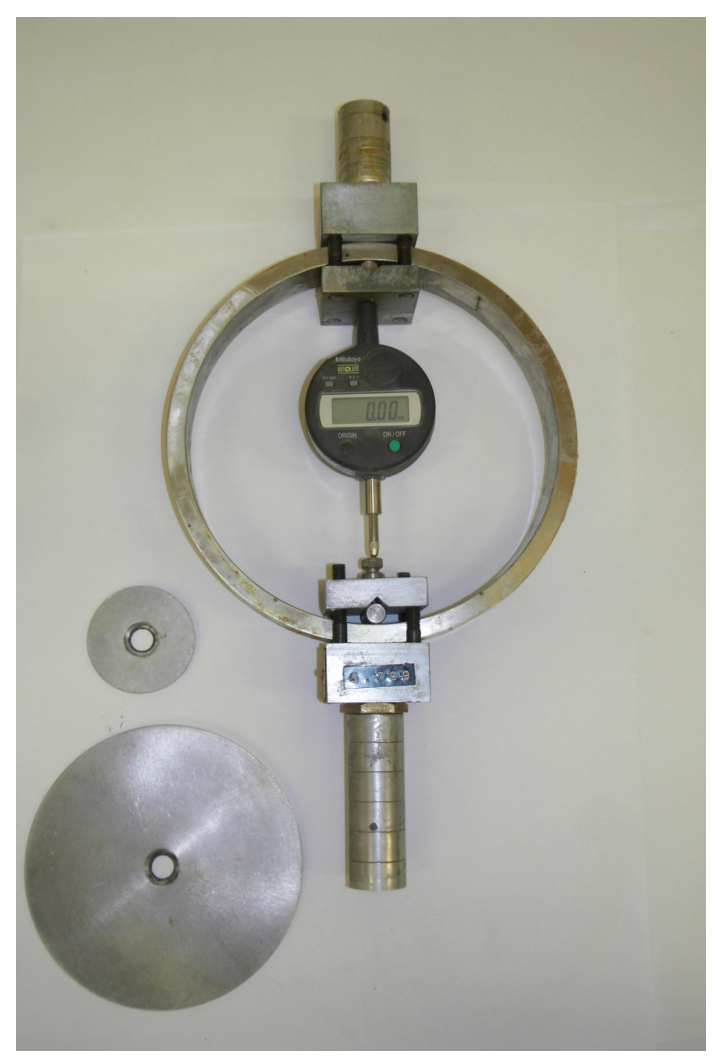

(b) Load cell used to record pressure readings

Figure 8: Pressure sinkage apparatus. 
surement and resin impregnation. As with the low and medium DST densities, it was not possible to perform measurements on the actual test samples, as this would result in disturbances to the particulate structure. As such, calibration samples were prepared specifically to obtain density measurements. In the case of ES-1 the volume of the sample prepared in the $64 \mathrm{~L}$ hard plastic bin was measured. The bin volume was measured by filling with water and measuring the mass. The density of water at room temperature was used to determine the volume. This method was necessary over resin impregnation due to the fine nature of the nepheline material. While it would be expected that the capillary action which ordinarily draws the resin through the material would be improved by such a material, this was not the case. The high viscosity of the resin did not allow the small voids to absorb the liquid, resulting in a clogging action. While this may have been possible to rectify with further diluting, it is likely even greater elongation of the resulting resin solid would occur.

Conversely, it was not possible to conduct a volumetric measurement of the $205 \mathrm{~L}$ drums used in the PST due to the excessive resulting mass of water. Here a resin impregnation technique is used. Developed at the University of Surrey, it is a method that allows the determination of the in-situ density of a loose, granular soil while causing minimal disruption to a prepared sample. This technique was detailed and later updated by [34], and further refinements for fine grained cohesionless materials were proposed by [35]. This test makes use of an epoxy resin containing Stycast W-19 resin, Catalyst 24LV, and the diluting agent Silquest A-1100. The optimal ratio of each chemical varied depending upon the particle size range, however, the nominal ratio used is 100 parts resin to 30 parts catalyst to 20 parts dilutant. Needles were manufactured $40 \mathrm{~cm}$ in length with a $2.1 \mathrm{~mm}$ outer diameter and a $1.6 \mathrm{~mm}$ inner diameter. The tips are bevelled at 45 degrees. Thin wire wool is inserted into the tip of the needle up to $25 \mathrm{~mm}$ to prevent the backfill of soil into the needle as it is inserted into the sample. 
The needles are attached to syringe reservoirs and inserted into the soil mass, as shown in Figure 9b. A known quantity of resin is dripped slowly into a syringe reservoir and allowed to flow under gravity through the needle into the soil until no further resin is absorbed. Resin losses are determined as the difference between pre- and post-test weight measurements of all resin preparation and injection equipment. Curing takes between 12 and 48 hours, dependent on the dilutant quantity added, after which the resin sphere is removed from the sand mass and coated in additional resin to fill external voids and waterproof the surface. The sphere is weighed to determine the mass and submerged in water to determine its volume using the Archimedes principle. The mass of the impregnated resin is subtracted from the mass of the sphere and the overall density of the soil alone was determined as follows:

$$
\text { Density of soil }=\frac{\text { mass of sphere }- \text { mass of resin }}{\text { volume of sphere }}
$$

The low, medium and high density measurements of ES-1 and ES-3 samples used for PST are shown in Table 6. The results indicate that ES-1 has an average low density similar to that of the medium density ES-1 DST samples shown in Table 2. This would be expected due to the height from which the simulant is poured and the self-compaction of the sample towards the bottom of the bin due to the soil surcharge. It is noted that the medium density range still fits well in between the lowest and highest density. It is also not unexpected that the high density value would be lower than the high density value from the DST as the PST equipment did not allow for the samples to be compacted. The range of these values is large enough to show variation in pressure-sinkage bearing strength as compaction level changes. It should also be noted that there is no standard deviation for the results of the high density test as the same quantity of material was used in each density measurement.

The resin impregnation technique used on ES-3 samples provided measurements for 


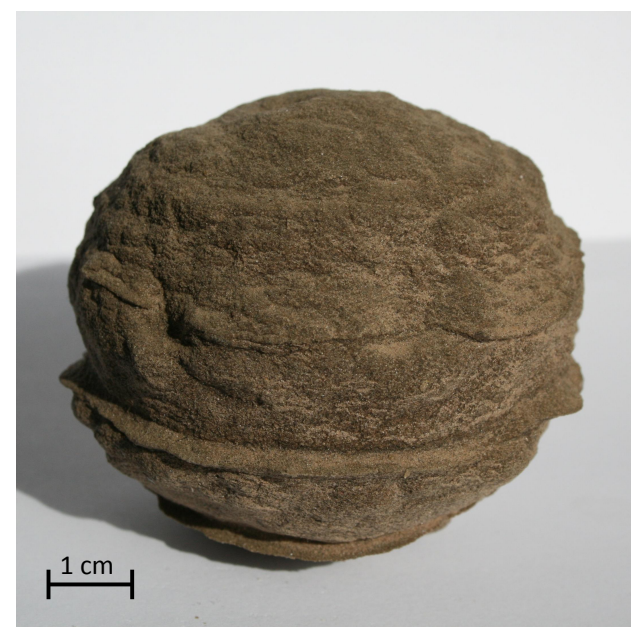

(a) The resulting sphere (SSC-2) [29]

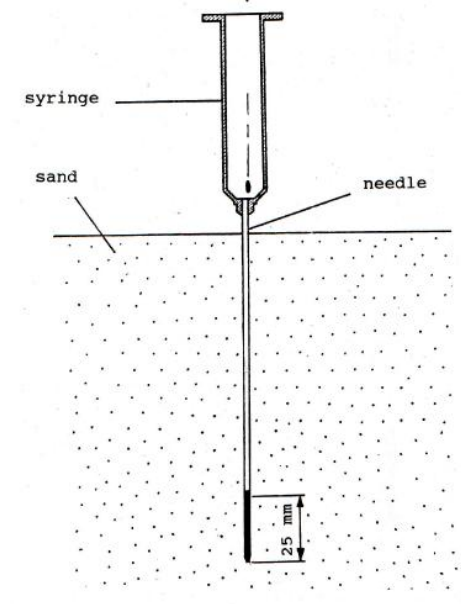

(b) Resin impregnation needle arrangement [35]

Figure 9: Pressure sinkage apparatus.

high, medium and low density samples. However, the resin impregnation conducted on ES-3 gave unexpected results: the low density $\left(1.66 \mathrm{~g} / \mathrm{cm}^{3}\right)$ is greater than the medium density $\left(1.61 \mathrm{~g} / \mathrm{cm}^{3}\right)$. The medium density is obtained by first preparing a poured density sample and the vibrating it. The vibration process to compact to medium density gave a clear indication of success by the observation of a drop in the surface level of the sample. Therefore, the anomalous result indicated by the resin impregnation has to be identified. To do so, $13 \mathrm{~L}$ samples of ES-3 were prepared using both vibration and pouring techniques and the density measured volumetrically.

The densities obtained from the vibrated sample in the $13 \mathrm{~L}$ bins are close to that obtained via resin impregnation in the drums with a good level of confidence, thus validating the high density resin impregnation measurements. The poured density value of $1.51 \mathrm{~g} / \mathrm{cm}^{3}$ obtained in the $13 \mathrm{~L}$ bin clearly indicates that the ES-3 low density value, obtained via resin impregnation, is anomalous. However, it is not accepted that the $13 \mathrm{~L}$ 
bin value can be used directly to determine the PST density value, as compaction due to the weight of ES-3 itself is not factored in the calculation. To obtain a better estimate of the poured PST sample density, the change in volume of poured ES-3 samples before and after compaction was considered. The drop in sample height after vibration was used to estimate the volumetric change between low and medium density samples. A low density estimate can be calculated by adjusting the medium density measurement for the volumetric change. The $13 \mathrm{~L}$ test results and refined density values for ES-3 are shown in Table 7. This final low density value is closer to that expected when considering the DST measurements.

To explain the anomalous results obtained via resin impregnation on poured ES-3 PST samples we have to consider the sensitivity of the localised compaction level around the needle as it is inserted. Furthermore, the high void ratio in the poured samples places a higher bias on the resin flow easily due to gravity, rather than by capillary action alone. The result of this can be seen in Figure 10a, where long, rod shaped ES-3 resin samples were formed instead of the more symmetric spheres obtained in previous tests [28] [29] [35]. The result of this phenomenon is a density measurement representative of a large depth range of ES-3, rather than a localised region of the sample.

\subsubsection{Results}

There are no approved standard procedures for pressure-sinkage test methods (such as the ASTM standards followed in other tests) or the analysis of the resulting data. Here, the method proposed by Wong [36] is used. What we aim to obtain is a linear best fit to a $\log$-log plot of the pressure sinkage data in order to determine the mechanical parameters used in the Bekker pressure-sinkage relationship, equation 2, as illustrated in Figure 11 . The Wong method is based on this concept, however, in practice it is an entirely analyt-

ical approximation method based on a least squares fit. Due to the resulting bias on low 


\begin{tabular}{l|ccc} 
& Low density $\left(\mathrm{g} / \mathrm{cm}^{3}\right)$ & Medium density $\left(\mathrm{g} / \mathrm{cm}^{3}\right)$ & High density $\left(\mathrm{g} / \mathrm{cm}^{3}\right)$ \\
\hline \hline ES-1 & & & 0.90 \\
Density & 0.79 & 0.82 & - \\
Std. dev. & 0.018 & 0.004 & 1.73 \\
ES-3 & & & 0.038 \\
Density & 1.66 & 1.61 &
\end{tabular}

Table 6: Average density of ES-1 and ES-3 when prepared using low, medium and high density sample preparation techniques for pressure-sinkage testing.

Low density $\left(\mathrm{g} / \mathrm{cm}^{3}\right) \quad$ Medium density $\left(\mathrm{g} / \mathrm{cm}^{3}\right) \quad$ High density $\left(\mathrm{g} / \mathrm{cm}^{3}\right)$

\begin{tabular}{|c|c|c|c|}
\hline \multicolumn{4}{|c|}{ Small scale tests } \\
\hline Density & 1.51 & - & 1.73 \\
\hline Std. dev. & 0.003 & - & 0.006 \\
\hline \multicolumn{4}{|c|}{ Final densities } \\
\hline Density & 1.58 & 1.61 & 1.73 \\
\hline Std. dev. & - & 0.019 & 0.038 \\
\hline
\end{tabular}

Table 7: Average density of ES-3 when prepared using low and high density sample preparation techniques for pressure-sinkage testing and tested using the volumetric technique. 


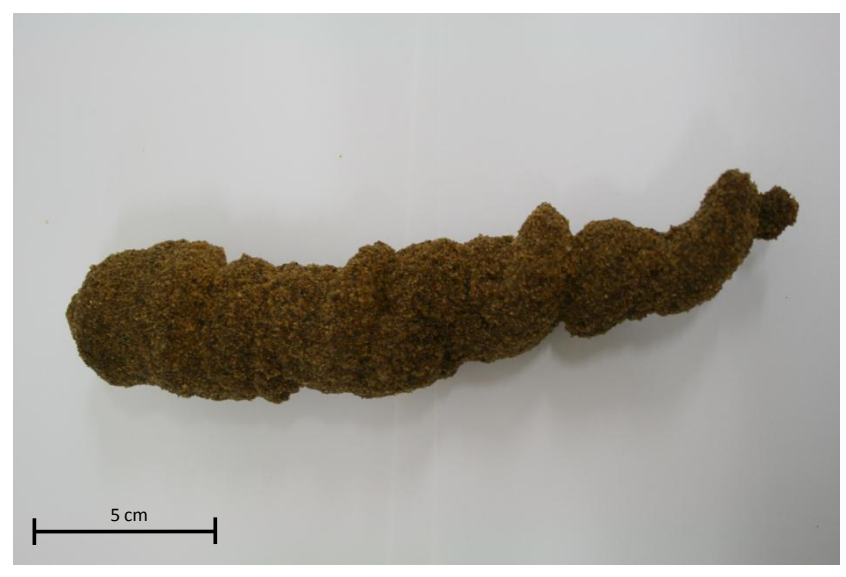

(a) Resin rod (ES-3)

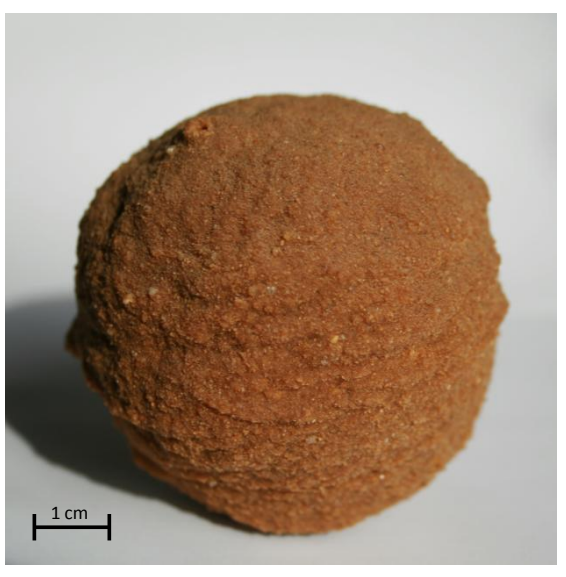

(b) Resin sphere (SSC-1) [28]

Figure 10: Simulant samples after resin injection

pressure values when using a log scale, a weighting factor is used to mitigate the issue. The value of $n$ is obtained from the average of that measured for each of the two plate sizes. Equation 2 is rearranged below to allow the remaining soil constants, $k_{C}$ and $k_{\phi}$, to be determined by the simultaneous equations 4 and 5 . This method makes use of the pressure readings $a_{1}$ and $a_{2}$ at $\log (z)=0$ and the plate sizes $b_{1}$ and $b_{2}$

$$
\begin{aligned}
& k_{c}=\frac{a_{2} b_{2}-a_{1} b_{1}}{b_{2}-b_{1}} \\
& k_{\phi}=\frac{a_{1}-a_{2} \cdot b_{1} b_{2}}{b_{2}-b_{1}}
\end{aligned}
$$

This method is developed in [9] to utilise a computer processor in order to mitigate ambiguity in the linear fits inherent in the trend lines produced by a human operator. The weighted least squares method provides the statistical best fit for the data. The method is applied here for the analysis of the ES-1 and ES-3 data using a modern desktop computer. For each soil and density, the data for each plate-pair test was cross-processed and averaged to produce the respective parameters. Table 8 presents the pressure-sinkage constants 


\begin{tabular}{l|ccc} 
& $n(-)$ & $k_{C}\left(\mathrm{kPa} / \mathrm{m}^{n}\right)$ & $k_{\phi}\left(\mathrm{kPa} / \mathrm{m}^{n-1}\right)$ \\
\hline \hline ES-1 & & & \\
Low density $\left(\mathrm{g} / \mathrm{cm}^{3}\right)$ & 0.67 & 67.28 & 0.68 \\
Medium density $\left(\mathrm{g} / \mathrm{cm}^{3}\right)$ & 0.71 & 61.96 & 1.30 \\
High density $\left(\mathrm{g} / \mathrm{cm}^{3}\right)$ & 0.75 & 142.36 & 1.66 \\
ES-3 & & & \\
Low density $\left(\mathrm{g} / \mathrm{cm}^{3}\right)$ & 0.92 & 1727.51 & -14.12 \\
Medium density $\left(\mathrm{g} / \mathrm{cm}^{3}\right)$ & 0.87 & 1931.13 & -16.41 \\
High density $\left(\mathrm{g} / \mathrm{cm}^{3}\right)$ & 0.76 & 2312.59 & -30.10
\end{tabular}

Table 8: Pressure-sinkage test results for samples of ES-3 prepared under low, medium and high density conditions.

for low, medium and high density samples of both ES-1 and ES-3. The results for tests in low, medium and high density samples of ES-1 are shown on a linear scale in Figure 12 and ES-3 in Figure 13, with Bekker model fits.

\section{Discussion}

\subsection{Direct Shear Testing}

The critical state shear stress is often considered in terramechanics as it is more applicable to the large strain conditions imparted by vehicles on the terrain. However, assuming a constant cohesion is not always valid, as moving outside of a tested range of normal stresses may result in a line of a different slope. While this has not been observed in the tangential analysis in general here, the values must be assumed as indicative and not taken as absolute readings for the simulant materials.

In general the distribution observed between each initial sample density in the ES-2 


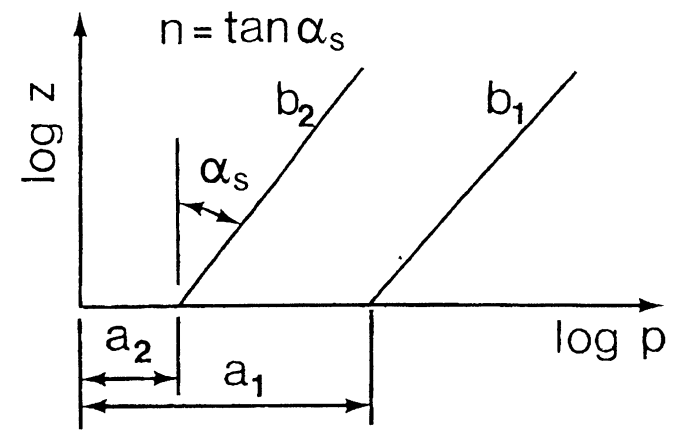

Figure 11: Graphical representation for determining the pressure readings, $a_{1}$ and $a_{2}$, for plate widths $b_{1}$ and $b_{2}[9]$

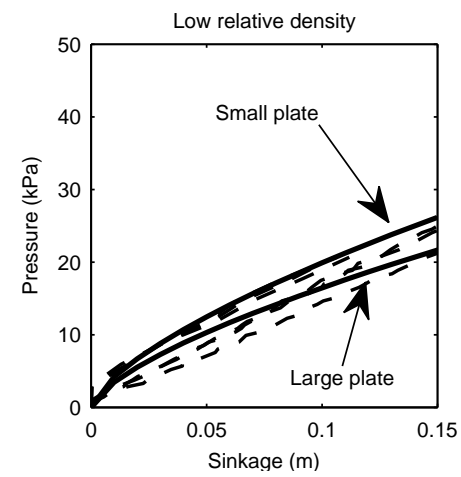

(a) Low density

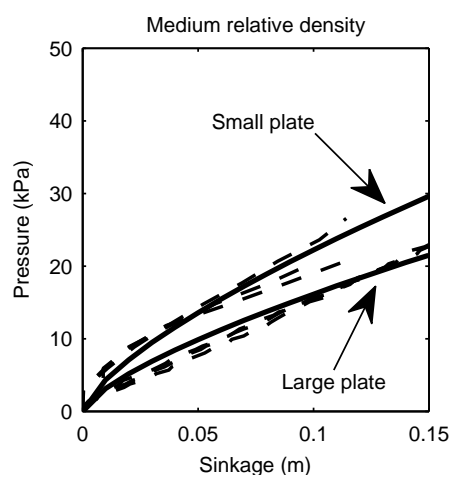

(b) Medium density

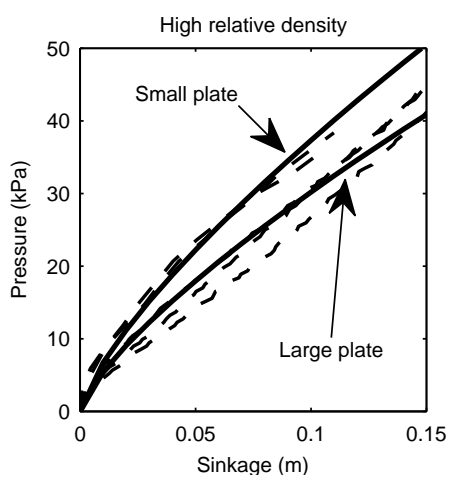

(c) High density

Figure 12: Pressure-sinkage tests for samples of ES-1. (Dashed line: experimental data; solid line: Bekker model fit for small plate) 


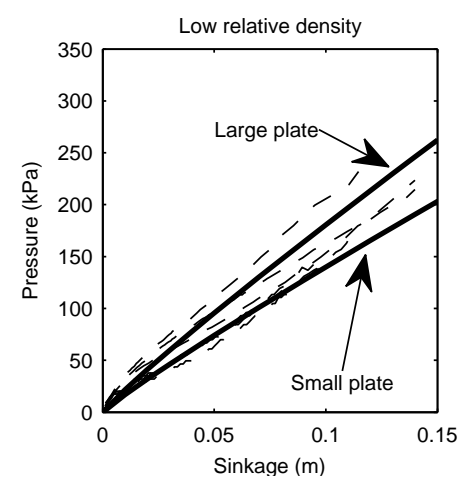

(a) Low density

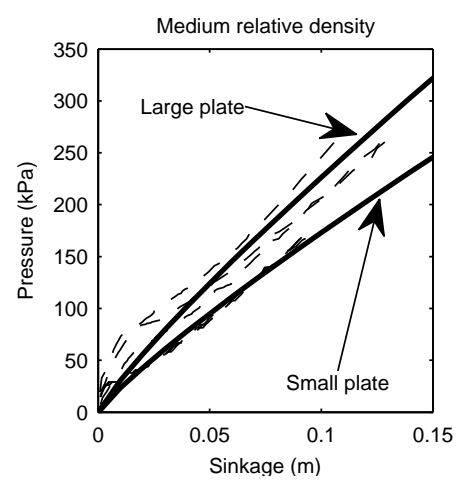

(b) Medium density

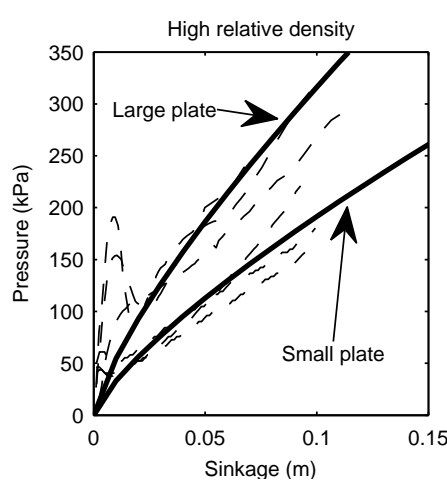

(c) High density

Figure 13: Pressure-sinkage tests for samples of ES-3. (Dashed line: experimental data; solid line: Bekker model fit for small plate)

and ES-3 shear strength measurements are consistent, regardless of the initial density conditions. This form can be seen in Figures $7 \mathrm{~b}$ and $7 \mathrm{c}$ and is appropriate when considering the critical state model for shear strength. The data show a consistent trend in the failure envelope, giving average instantaneous friction angles of 39.3 degrees and 35.9 degrees for ES-2 and ES-3 respectively. The ES-2 particle shape has been observed as more angular than ES-3 particles, therefore it is not unexpected to find ES-2 producing a friction angle over that of ES-3.

An exception to these findings is seen in the DST results for ES-1. The data produce an unusual plot where the high density shear stresses are consistently higher than those measured at low and medium density, which follow a consistent trend in shear strength as expected by taking measurements at the critical state of shearing. The friction angle appears to be similar to that of the lower and medium density materials; however, the apparent cohesion is significantly and consistently higher, implying these are not just anomalous results. The shear plots also indicate strain-hardening is occurring in the ES-1 samples, as seen in Figure 6a (all tests on ES-1 followed this trend). This is an indicator 
of shearing in dense, granular material where limited or non-flow deformation is observed [37]. It is hypothesised by the authors that the particle shape may be having an effect due to the tight compaction . This result appears to illustrate the limitation of critical state theory in certain materials. Similar results have been shown in [38]. Here a new type of soil behaviour has been observed which does not obey the critical state model. It is suggested that ES-1 is also demonstrating this transitional behaviour. This is similar in manner to cohesive soils, also typically platy in nature, which do not follow critical state theory. It would be expected to find a polished shear surface as the particles align due to the nature of their shape, which is also wavy in form due to the nature of the shear plane development in the DST of platy materials. Both these effects appear to be the case in the ES-1 sample removed from the shear box after DST, shown in Figure 14a. Similar formations in the shear surface have been observed in similar tests on other platy materials. In [39] direct shear tests were interrupted at successive displacements and photographs taken of sections of each sample. Two such sections are reproduced in Figure $14 \mathrm{~b}$ illustrating the shear plane form at the critical state. A similar result is presented in [40], where sand-mica mixes of varying proportions are subjected to DST. The findings show that at $40 \%$ mica fraction a split shear zone is formed with slickensided texture to the surface. Analysis of electron microscope images of the shear plane show clay particles orientated parallel to the surfaces of the sand grains.

This would suggest that if the particle shape of ES-1 is not rounded, as generally assumed in granular or "cohesionless" materials, the greater specific surface area could cause the observed higher levels of apparent cohesion. In finer particulate material (such as ES-1), inter-particle forces such as electrostatics, which has been observed in the material samples, or Van der Waals forces between these extremely small particles begin to have a notable effect on cohesion. Furthermore, despite heating the material at $110^{\circ}$ for 48 hours the cohesive nature of the material is still observable, implying some water molecules may 


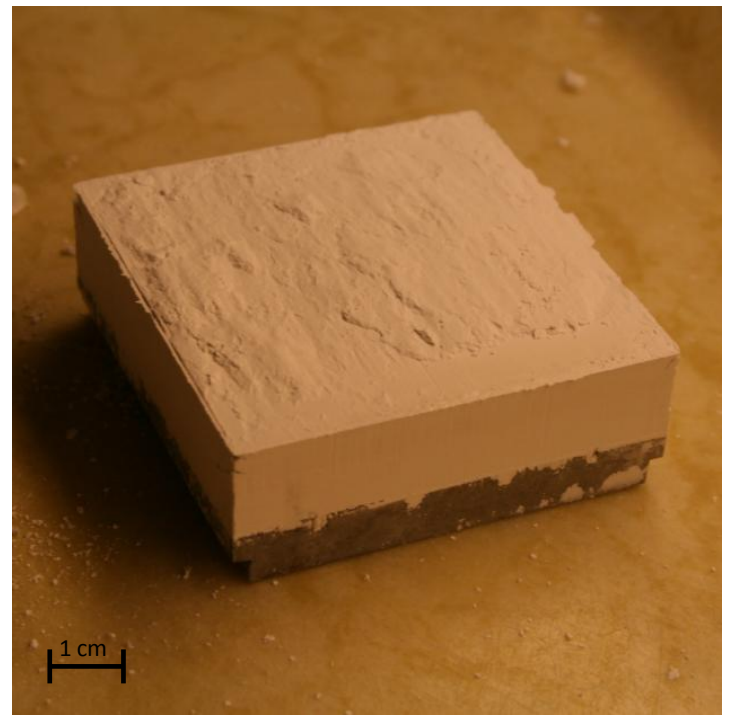

(a) Here the sample is seen to hold its form after removal from the shear box and shows the wavy form of the shear plane.
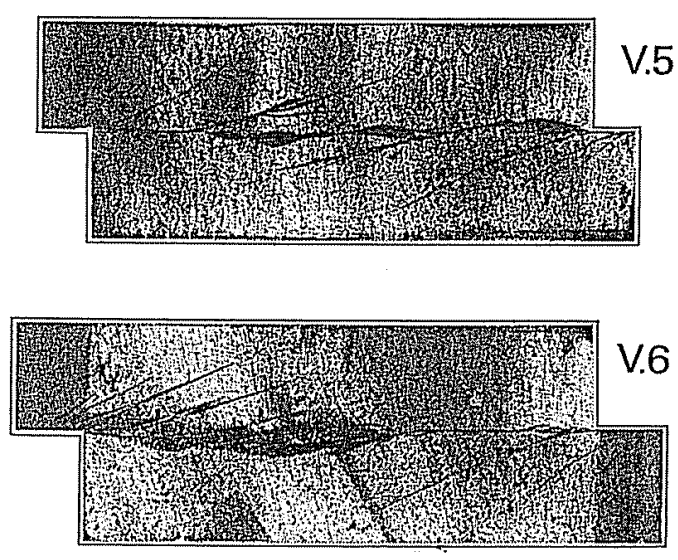

(b) A similar process is described in [39]

Figure 14: Post-DST analysis of ES-1 sample.

still be bound to the particle surfaces. It would require further heating at a much higher temperature to assess whether this is the case or if the response is caused by something else (such as the angular particles interlocking under compression). The unanticipated nature of the results will be explored in a future investigation into the behaviour of this material.

\subsection{Pressure Sinkage Testing}

It is clear in the case of these results that the initial density of the simulant has a measurable effect on the final parameter values. The values for measurements on various sands and sandy loams, similar to those used here, have also shown similar results, including negative $k_{c}$ values, as reported in [9] [41]. Fine granular materials are also subjected to PST in [42] using a similar experimental method. However, the analysis procedure utilises the data fitting routines included in the MATLAB software package. 
Unlike the DST parameters, the parameters measured for ES-1 and ES-3 all indicate a sensitivity to the initial density of the sample, shown in Figure 15 . We see a downward trend in the soil exponent, $n$, with density in ES-3 and an upward trend in ES-1. A similar pattern is observed in the cohesion coefficient, $k_{C}$, and a consistent upward trend in the internal friction angle coefficient, $k_{\phi}$, of both simulants. We can draw from these findings when analysing the performance of a rover on loose terrain using the terramechanics theory proposed by Bekker in [8]. Moreover, consideration of the initial conditions of the soil being used to test any vehicle must be included in the analysis.

The presence of an initial pressure peak in the first few centimetres of sinkage during the PST of ES-3 at high density, shown in Figure 13c was an unexpected finding in this study. As this peak does not reflect the response of the soil deformation alone the Bekker model does not account for this unexpected behaviour. Therefore, all data under $2 \mathrm{~cm}$ sinkage were rejected in the analysis of the ES-3 PST. It is interesting to note that these peaks are visible only in the high density tests. This is identical to the shearing response observed in the DST tests on the same material, indicating a peak in the shearing stress as the shear zones are first mobilised in the sample. It is also understood that peak shearing stress in DST is proportional to the sample density, which would explain the lack of peak stresses in the lower density ES-3 PST [32]. However, Figure 16 shows the PST plot from an identical test performed on ES-3 in the smaller $64 \mathrm{~L}$ bin. Here we see an even greater initial pressure peak and post-peak residual pressure that, despite the efforts of the authors, would indicate there is also interaction with the container boundaries.

Considering the experimental conditions for the PST in this article, there are a further two, more subtle aspects of the shearing process that are most likely to have contributed to the peaks effect. Firstly, the dilation in the material surrounding the shear plane may be constrained by the container walls. This will result in a greater stress within the initial turbulent zone of particles than that under unconstrained conditions. Furthermore, 


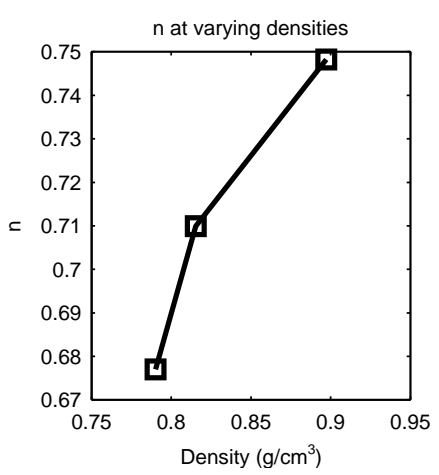

(a) ES-1 soil exponent

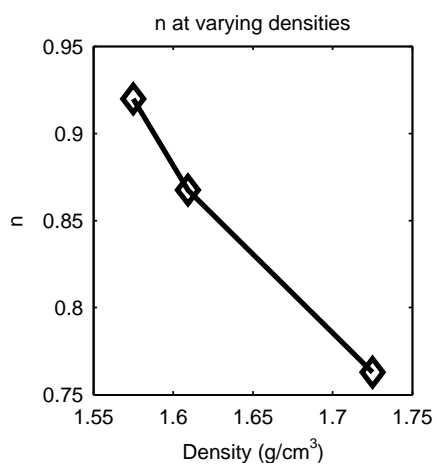

(d) ES-3 soil exponent

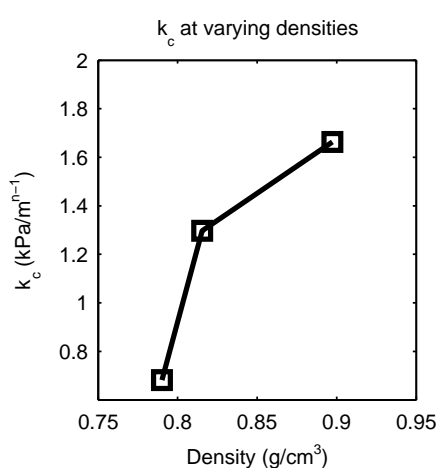

(b) ES-1 coefficient of cohesion

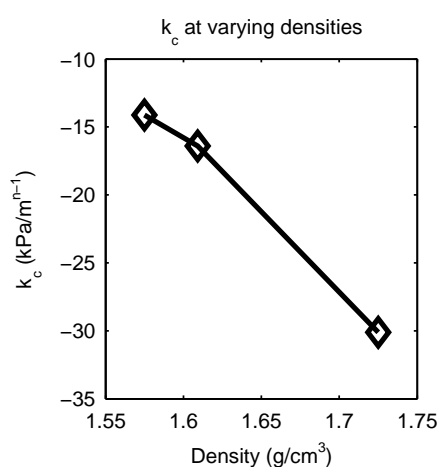

(e) ES-3 coefficient of cohesion

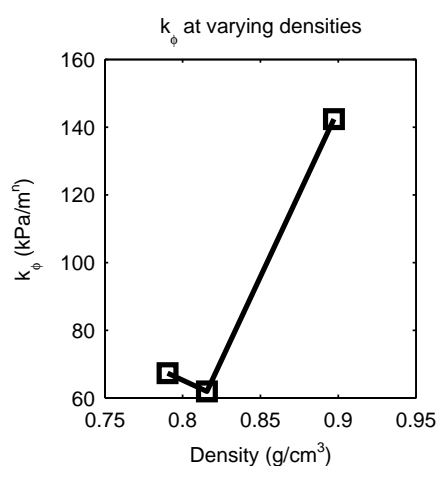

(c) ES-1 coefficient of internal friction

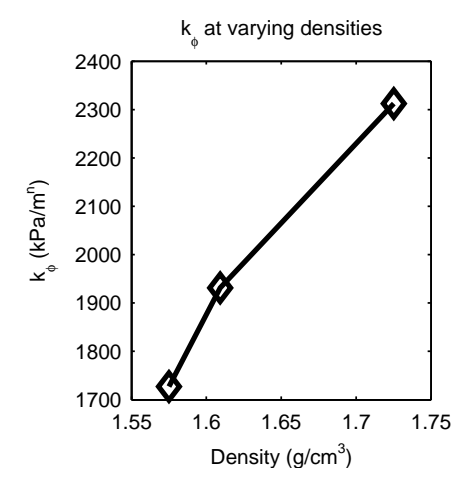

(f) ES-3 coefficient of internal friction

Figure 15: Mechanical parameters for the Bekker pressure sinkage model 


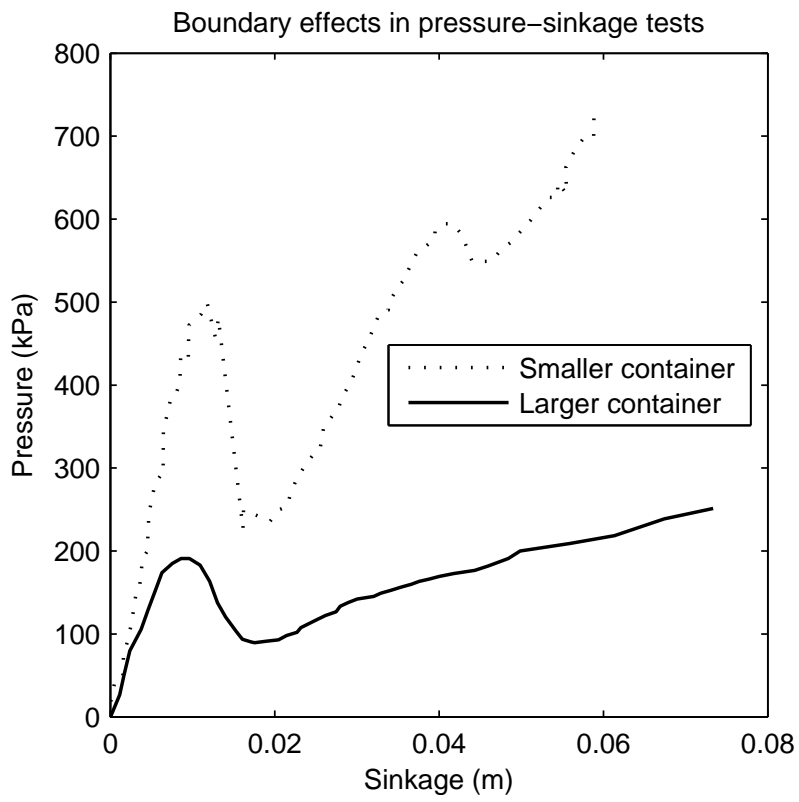

Figure 16: Peak shear force measurements for high density ES-3 in two containers of different diameter. 


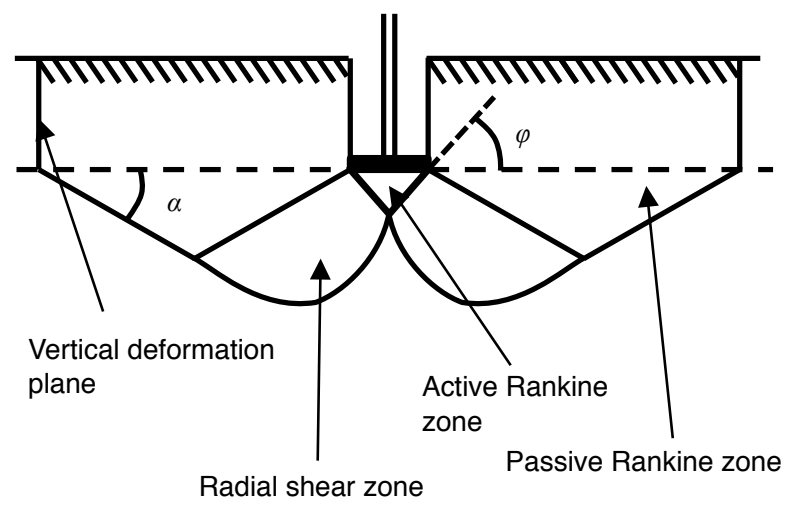

Figure 17: Shear planes developed beneath a pressure plate in a deformable soil sample

if the turbulent zone does not contract beyond the constraining limits of the boundaries as shearing continues, it will be unable to settle into a completely homogenous shearing plane. Consequently, this will result in a higher residual stress, such as that observed in the smaller container. Secondly, the shear planes developed by this process are defined by [43] as the combination of active and passive Rankine zones connected by a radial logarithmic spiral. This is illustrated in Figure 17 . The extent of the reach of the shear planes are proportional to the pressure plate diameter and internal friction angle $(\alpha=45-\phi / 2)$. It is predicted that a larger pressure plate will extend the range of the shearing planes, resulting in greater interaction with the container boundaries. Again, this is seen in the high density plot in Figure 13c, where the magnitude of the peaks in the small plate tests is much lower than that of the larger plate tests. These shear planes are also sensitive to the internal friction angle, as seen from the ES-1 tests in the same, $64 \mathrm{~L}$ container. This material has a friction angle lower than that of ES-3, but does not have any such initial peaks in the pressure. 


\section{Conclusion}

This paper has presented the direct shear tests (DST) and the pressure sinkage tests (PST) performed on three new Martian soil simulants, proposed as test materials for the traction performance testing of the ExoMars rover locomotion system. Each soil simulant was tested at three levels of density to better understand how the macro-mechanical properties of soil (specifically cohesion, friction angle, and pressure-sinkage constants) change under varying conditions. Unlike ES-2 and ES-3, the critical shear strength of ES-1 does appear to depend on its initial density. This is in contradiction with the critical state theory, observed in practice in the two other simulants. Further experimental exploration of the behaviour of ES-1 is planned.

The PST on ES-3 exhibited unexpected initial peaks in the first $2 \mathrm{~cm}$ of sinkage; this was observed in the high density samples in particular. However, these were not seen in the tests on ES-1. The suggested cause is the boundary effects resulting from the use of a container of insufficient size. The lower internal friction angle of ES-1 is likely to have mitigated this effect in identical tests using the same equipment.

The three new Martian soil simulants defined here have been subjected to accepted and proven methods of mechanical testing. These parameters allow both the comparison of the simulants to their Martian counterparts and their use in bulk quantities when creating terrain analogues terrestrially. Furthermore, the tests performed on these materials are extensive and thorough, illustrating the measurable variations in their respective parameters and identifying preliminary trends in the values. These effects have also allowed the identification of potential pitfalls in the measurement of the parameters, where boundary effects may influence the instrument readings. Careful consideration of the preparation of simulant test samples during the development of exploration rover technology is necessary to ensure the tractive performance is fully and accurately profiled. 


\section{Acknowledgements}

The work described in this paper was performed under a direct contract from EADS Astrium within the ESA ExoMars project for which Astrium is the prime contractor for the ExoMars rover vehicle and Thales Alenia - Italy is the ExoMars mission prime. The authors would like to thank the project teams involved with this study at Astrium, Thales and ESA.

\section{References}

[1] N. Patel, R. Slade, J. Clemmet, The ExoMars rover locomotion subsystem, Journal of Terramechanics 47 (4) (2010) 227-242.

[2] USDA, Soil Survey Manual, US Department of Agriculture, 1993.

[3] H. Durgunoglu, J. Mitchel, Influence of penetrometer characteristics on static penetration resistance. I-Analysis. II-Evaluation of theory and implications for practice, in: Specialty conference on in-situ measurement of soil parameters, 172-189, 1975.

[4] N. Patel, An investigation into optimal mobility system for planetary rovers, Ph.D. thesis, University of Surrey, 2005.

[5] G. Scott, C. Saaj, An investigation into the the trafficability of a low mass legged rover on loosely packed granular soils, in: Proceedings of the 2009 International Society for Terrain and VehicleSystems (ISTVS) European Conference, -, 2009.

[6] J. Hutchinson, T. Wu, Advances in applied mechanics, no. v. 24 in Advances in Applied Mechanics, Academic Press, ISBN 9780120020249, 1984.

[7] C. Clayton, M. Matthews, N. Simons, Site investigation, Blackwell Science, Oxford, 2nd edn., URL wWw . geotechnique . info, 1995. 
[8] M. G. Bekker, The Theory of Land Locomotion, The University of Michigan Press, 1956.

[9] J. Wong, Data processing methodology in the characterization of the mechanical properties of terrain, Journal of Terramechanics 17 (1) (1980) 13-41.

[10] K. Seiferlin, P. Ehrenfreund, J. Garry, E. Gunderson, K.and Hütter, G. Kargl, A. Maturilli, J. Merrison, Simulating Martian regolith in the laboratory, Planetary and Space Science 56 (15) (2008) 2009-2025.

[11] H. Perko, J. Nelson, J. Green, Mars soil mechanical properties and suitability of Mars soil simulants, Journal of Aerospace Engineering 19 (2006) 169.

[12] G. Scott, C. Saaj, Measuring and Simulating the Effect of Variations in Soil Propertieson Microrover Trafficability, in: Proceedings of the American Institute of Aeronautics and Astronautics, 1801 Alexander Bell Dr., Suite 500 Reston VA 20191-4344 USA, -, 2009.

[13] H. Oravec, X. Zeng, V. Asnani, Design and characterization of GRC-1: A soil for lunar terramechanics testing in Earth-ambient conditions, Journal of Terramechanics 47 (2010) 361-377.

[14] G. Peters, W. Abbey, G. Bearman, G. Mungas, J. Smith, R. Anderson, S. Douglas, L. Beegle, Mojave Mars simulant-Characterization of a new geologic Mars analog, Icarus 197 (2) (2008) 470-479.

[15] W. Carrier III, L. Bromwell, R. Torrence Martin, Strength and compressibility of returned lunar soil, in: Lunar and Planetary Science Conference Proceedings, vol. 3, $3223,1972$. 
[16] W. Carrier III, Lunar Soil Simulation and Trafficability Parameters, Lunar Geotechnical Institute, Tech. Rep - (2006) -.

[17] D. Cornforth, Prediction of drained strength of sands from relative density measurements, Evaluation of Relative Density and Its Role in Geotechnical Projects Involving Cohesionless Soils-STP 523 ASTM STP 523 (1973) 281.

[18] M. Al-Hussaini, Influence of Relative Density on the Strength and Deformation of Sand under Plane Strain Conditions, Evaluation of Relative Denisty and Its Role in Geotechnical Projects Involving Cohesionless Soils ASTM STP 523 (1973) 332.

[19] G. Heiken, D. Vaniman, B. French, Lunar sourcebook: A user's guide to the Moon, Cambridge Univ Pr, 1991.

[20] J. Mitchell, W. Houston, R. Scott, N. Costes, W. Carrier III, L. Bromwell, Mechanical properties of lunar soil: Density, porosity, cohesion and angle of internal friction, in: Proceedings of the Lunar Science Conference, vol. 2, -, 1972.

[21] C. Brunskill, V. Lappas, The effect of relative soil density on microrover trafficability under low ground pressure conditions, in: Proceedings of the 2009 International Society for Terrain and Vehicle Systems (ISTVS) European Conference, -, 2009.

[22] A. El Shafie, R. Ulrich, L. Roe, Penetration Forces for Subsurface Regolith Probes, in: Lunar and Planetary Institute Science Conference Abstracts, vol. 40, 1205, 2009.

[23] T. P. Gouache, Y. Gao, P. Coste, Y. Gourinat, Lunar regolith simulant preparation and its impact on static penetration resistance and DRD penetration, in: European Planetary Science Congress, Rome, Italy, -, 2010. 
[24] T. P. Gouache, Y. Gao, P. Coste, Y. Gourinat, Experimental parametric evaluation of dual-reciprocating drilling mechanism performance, in: European Conference on Spacecraft Structures, Materials and MechanicalTesting, Toulouse, France, -, 2009.

[25] R. Arvidson, J. Bell III, P. Bellutta, N. Cabrol, J. Catalano, J. Cohen, L. Crumpler, D. Des Marais, T. Estlin, W. Farrand, et al., Spirit Mars Rover Mission: Overview and selected results from the northern Home Plate Winter Haven to the side of Scamander crater, Journal of Geophysical Research 115 (2010) -, ISSN 0148-0227, URL http: //dx.doi.org/10.1029/2010JE003633.

[26] S. Upadhyaya, Advances in soil dynamics, American Society of Agricultural Engineers, ISBN 0929355520, 1994.

[27] ASTM, Standard Test Methods for Laboratory Determination of Water (Moisture) Content of Soil and Rock by Mass, 2005.

[28] T. P. Gouache, C. Brunskill, G. P. S. Y. Gao, P. Coste, Y. Gourinat, Regolith simulant preparation methods for hardware testing, Planetary and Space Science 58 (14-15) (2010) 1977 - 1984, ISSN 0032-0633.

[29] G. P. Scott, Steps towards characterising legged microrover performance on cohesionless planetary soils, Ph.D. thesis, University of Surrey, 2009.

[30] J. Kolbuszewski, R. Jones, The preparation of sand samples for laboratory testing, Proceedings of the Midland Soil Mechanics and Foundation Engineering Society 4 (1961) 107-123.

[31] R. Craig, Craig's soil mechanics, Taylor \& Francis, 2004.

[32] D. W. Taylor, Fundamentals of Soil Mechanics, Chapman \& Hall, Limited, London, England, 6th edition edn., 1948. 
[33] G. Barnes, Soil Mechanics: Principles and Practice, Palgrave Macmillian, Basingstoke, England, 2nd edn., 2000.

[34] C. R. I. Clayton, A. V. D. Bica, S. R. Moore, A resin impregnation technique for the determination of the density variations in completed specimens of dry cohesionless soil, Geotechnique Vol. 44 No. 1 (1994) pp. 165-173.

[35] A. V. D. Bica, A study of free embedded cantilever walls in granular soil, Ph.D. thesis, University of Surrey, 1991.

[36] J. Wong, Theory of ground vehicles, Wiley-Interscience, 4th edn., 2008.

[37] D. Wanatowski, J. Chu, R. Lo, Strain-softening behaviour of sand in strain path testing under plane-strain conditions, Acta Geotechnica 3 (2) (2008) 99-114, ISSN 1861-1125.

[38] F. Altuhafi, B. Baudet, P. Sammonds, The mechanics of subglacial sediment: an example of new transitional behaviour, Canadian Geotechnical Journal 47 (7) (2010) 775-790, ISSN 0008-3674.

[39] N. Morgenstern, J. Tchalenko, Microscopic structures in kaolin subjected to direct shear, Geotechnique 17 (1967) 309-328.

[40] J. Lupini, A. Skinner, P. Vaughan, Drained residual strength of cohesive soils, Geotechnique 31 (1981) 181-213, ISSN 0016-8505.

[41] J. Wong, Terramechanics and Off-Road Vehicle Engineering, ButterworthHeinemann, 2010.

[42] M. Apfelbeck, S. Kuß, A. Wedler, B. Gibbesch, A. andRebele, B. Schäfer, A novel Terramechanics testbed setup for planetary Rover wheel-soil Interaction, in: Pro- 
ceedings of the 11th European Regional Conference of the International Society for Terrain-Vehicle Systems, -, 2009.

[43] K. Terzaghi, Theoretical Soil Mechanics, John Wiley and Sons, 3rd edn., 1943. 